\title{
Ischemic Cauda equina Syndrome Due to Spinal Embolization as a First Manifestation of an Infrarenal Abdominal Aortic Aneurysm
}

\author{
Youssef Zrihni1 $^{*}$, Tiia Kukkonen², Fabien Thaveau', Elie Girsowicz ${ }^{1}$, Yannick Georg1, \\ Nabil Chakfe ${ }^{1}$ \\ ${ }^{1}$ Department of Vascular Surgery and Kidney Transplantation, University Hospital of Strasbourg, Strasbourg, \\ France \\ ${ }^{2}$ Department of Vascular Surgery, Helsinki University Central Hospital, Helsinki, Finland \\ Email: ${ }^{*}$ Youssef.zrihni@hotmail.com
}

Received 21 April 2015; accepted 26 June 2015; published 29 June 2015

Copyright (C) 2015 by authors and Scientific Research Publishing Inc.

This work is licensed under the Creative Commons Attribution International License (CC BY).

http://creativecommons.org/licenses/by/4.0/

(c) (i) Open Access

\begin{abstract}
Spinal cord ischemia is an uncommon complication of an abdominal aortic aneurysm (AAA). We report the case of a 59-year-old man admitted for an acute ischemic Cauda equina syndrome secondary to a spinal cord embolization from an unknown partially thrombosed aortic aneurysm. The patient being at risk of further embolization, we achieved an emergency EVAR. The vascular post-operative course was uneventful. Neurologically, a post operative lumbar medullar MRI confirmed an ischemic Cauda equina syndrome and six months after the surgery, the patient still had a motor and sensory deficit in both lower limbs.
\end{abstract}

\section{Keywords}

Cauda equina Syndrome, Aortic Aneurysm, Endovascular Repair

\section{Introduction}

Complications of an abdominal aortic aneurysm (AAA) can include massive hemorrhage as a result of a rupture, ischemia by either thrombotic or embolic mechanisms, and urinary or venous compression. Spinal cord ischemia is an uncommon complication of an AAA, sometimes associated with thrombosis of the abdominal aorta. More often than as a first symptom of an AAA [1] [2], spinal cord ischemia occurs also in context with the different procedures performed for aortic aneurysms and others pathologies [3] [4].

"Corresponding author.

How to cite this paper: Zrihni, Y., Kukkonen, T., Thaveau, F., Girsowicz, E., Georg, Y. and Chakfe, N. (2015) Ischemic Cauda equina Syndrome Due to Spinal Embolization as a First Manifestation of an Infrarenal Abdominal Aortic Aneurysm. World Journal of Cardiovascular Surgery, 5, 58-62. http://dx.doi.org/10.4236/wjcs.2015.56010 
We report a patient with an AAA which initially presented as Cauda equina syndrome caused by embolization of the intraluminal thrombus to the spinal cord which was successfully treated by anticoagulation, spinal fluid drainage and emergent EVAR.

\section{Case Report}

A 59-year-old man was referred to our hospital emergency unit with acute motor and sensory deficits in both lower limbs. Cardiovascular risk factors were arterial hypertension and dyslipidemia. Clinical examination revealed a complete motor and sensory deficit with paresthesia in both lower limbs. His deep tendon reflexes and the Babinski sign were absent. The anal reflex was abolished with saddle block anesthesia and acute urinary retention. Vascular examination of both legs was normal with distal pulses felt.

Lumbar medullar magnetic resonance imaging (MRI) was normal but showed an infrarenal AAA (Figure 1). The finding was subsequently confirmed by a contrast enhanced CT scan (Figure 2). The aneurysm was partially thrombosed with a maximal diameter of $7.5 \mathrm{~cm}$ with no signs for rupture.

Laboratory investigations showed mild anemia and leukocytosis. Electrolytes and serum creatinine levels were within normal limits. Results of initial measurements of coagulation parameters, electrocardiogram, and chest radiographs were normal.

The initial management consisted of anticoagulation with heparin associated with spinal fluid drainage. As the clinical diagnosis was a spinal cord embolization from the aortic aneurysm with an ischemic Cauda equina syndrome, he was deemed to be at risk for further embolization. The patient was transferred to the operating theatre without delay and the aneurysm was repaired with an aortic endograft. The control angiogram showed successful exclusion of the infrarenal aortic aneurysm without evidence of an endoleak or distal embolization. The postoperative CT showed a total exclusion of the AAA without any sign of endoleak (Figure 3).

The immediate postoperative recovery was uneventful. Forty-four hours later he began to regain a little sensory and motor function to his legs. When another MRI was performed, this revealed spinal cord ischemia from the level of T10 to terminus cone (Figure 4).

At post-operative follow up after 6 months, the patient is still hospitalized in a reeducation neurologic center for neurologic rehabilitation. He still has a motor and sensory deficit in both lower limbs.

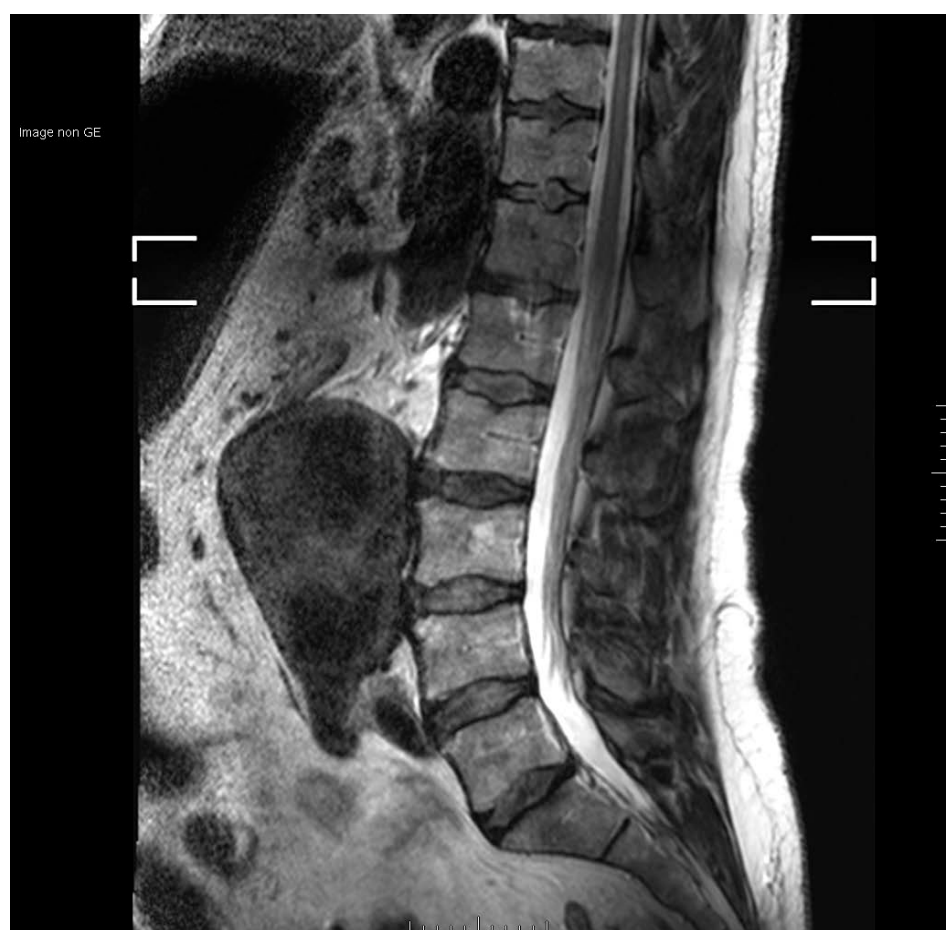

Figure 1. Spinal MRI-sagittal T2- normal spina equida signal. Aneurysm is easily seen as a hyposignal structure. 


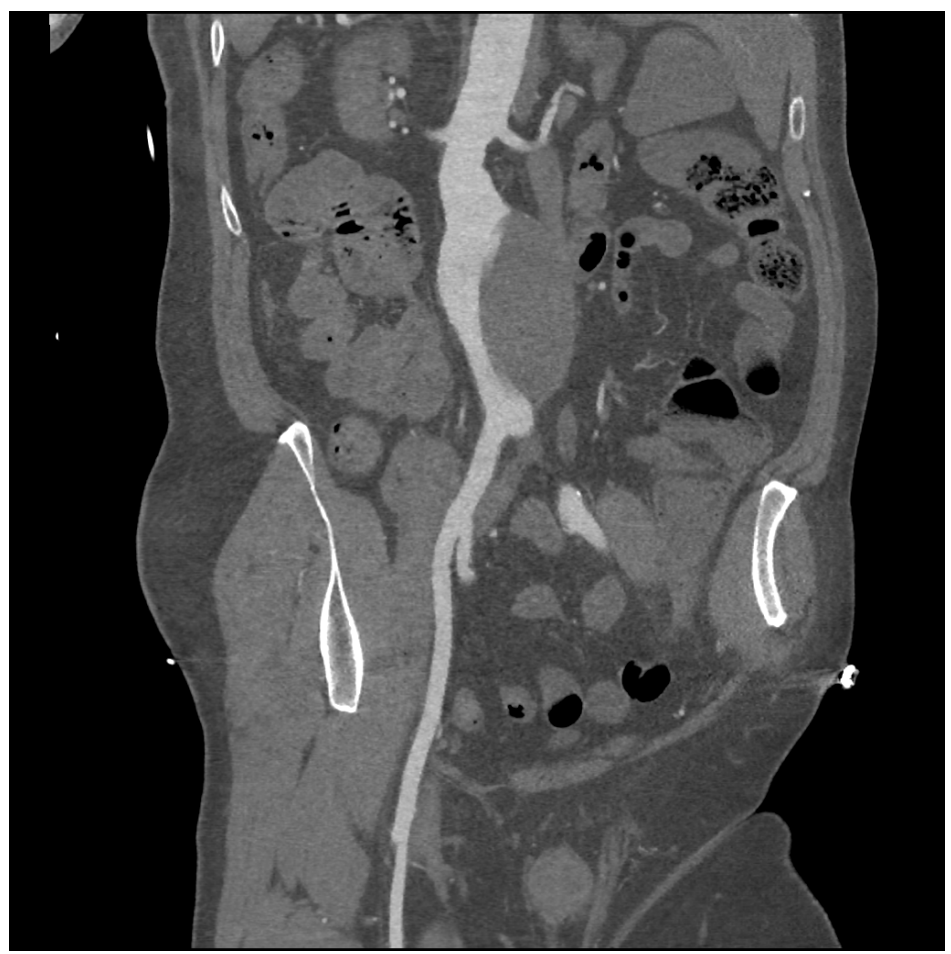

Figure 2. Pre operative CT scan with curvilinear reconstruction showing an infra-renal aneurysm with lateral thrombosis.

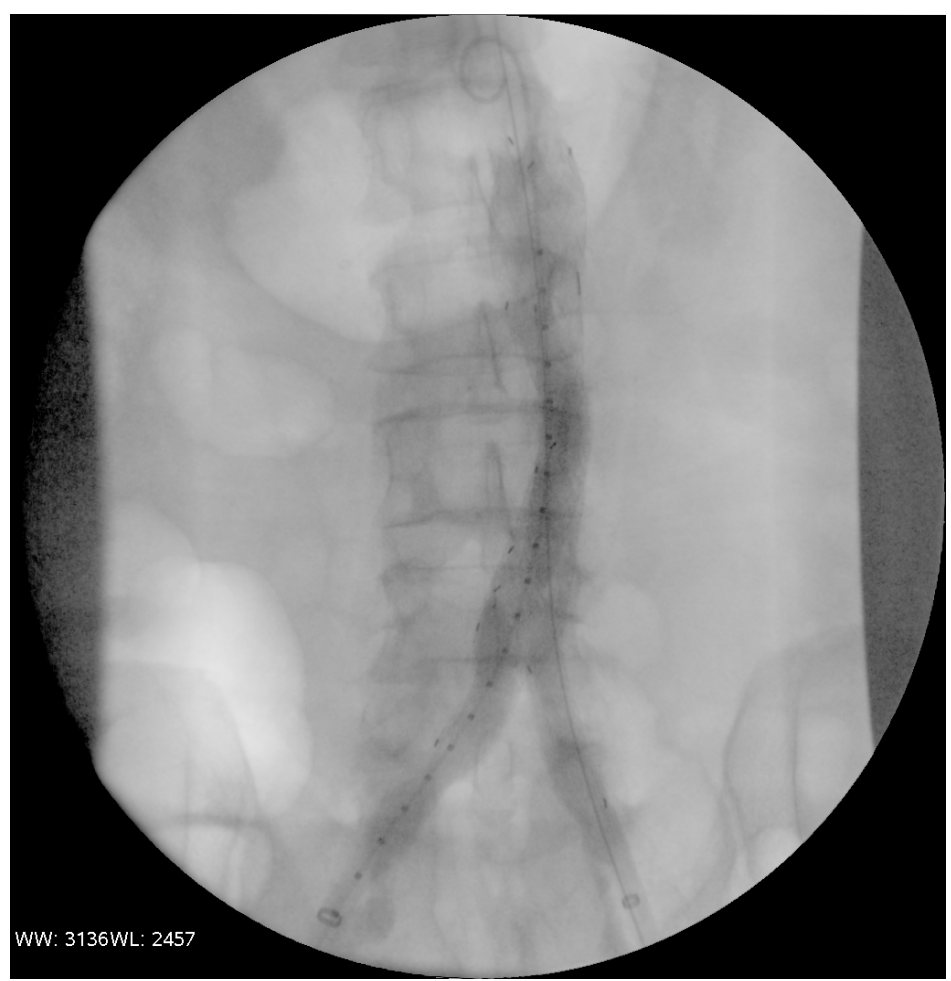

Figure 3. Per-operative control angiogram showing successful exclusion of the infrarenal aortic aneurysm without any endoleak or distal embolization (EVAR). 


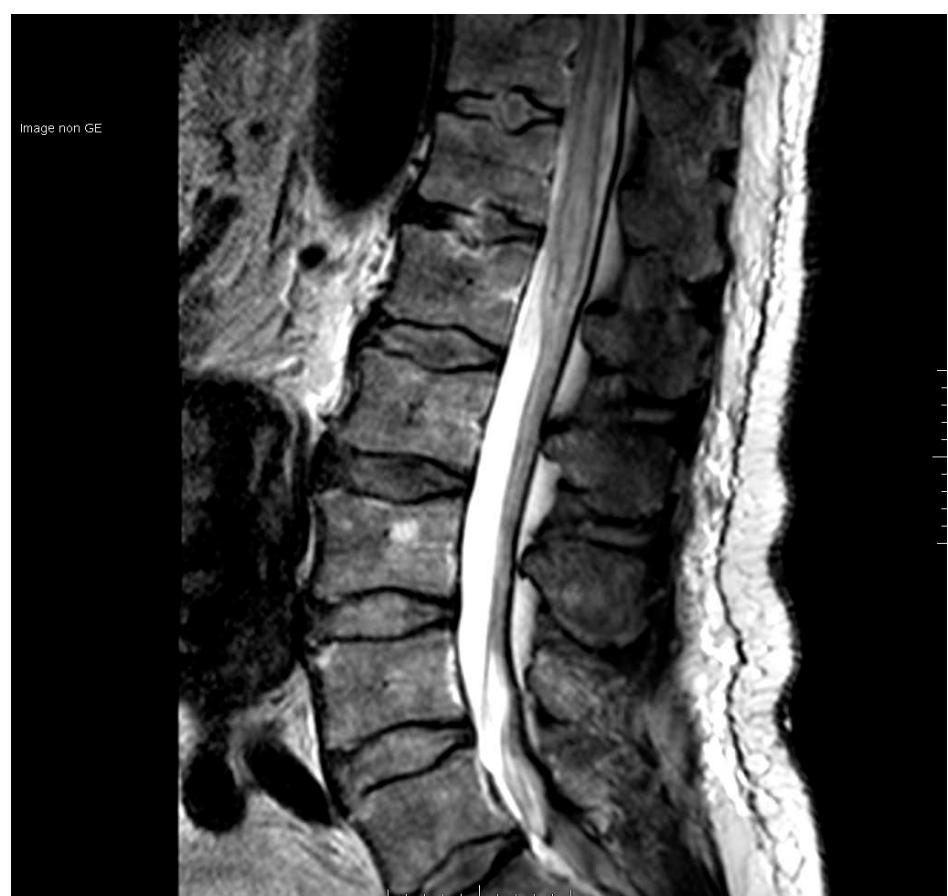

Figure 4. Post-operative lumbar MRI: apparition of a large T2 hypersignal confirming the Cauda equina syndrome.

\section{Discussion}

Cauda equina syndrome is characterized by motor and sensory deficits of the lower limbs with or without reflex change, saddle block anesthesia and bowel and/or bladder dysfunction [2]. Ischemic Cauda equina syndrome following an untreated AAA is a rare condition [1].

Several other associations of aortic aneurysms with spinal cord infarction have been described. The incidence of spinal cord infarction as a complication of aneurysm repair is $1 \%$ - $2 \%$ in all infrarenal aortic surgery [3] [5] [6]. A delayed neurologic deficit associated to spinal cord ischemia after elective repair of infrarenal AAA is also a known chain of events [3] [7]. Another well-known cause for ischemic Cauda equina syndrome is aortic dissection, the likely mechanism of spinal cord infarction being occlusion of the origins of major arteries feeding the spinal vasculature, such as the great anterior artery of Adamkiewicz at T10/12 [8]. Direct erosion by a large aneurysm of the lumbar vertebral bodies in the setting of rheumatoid arthritis, leading to spinal cord compression, has also been described [9].

In the literature, a case of an acute thrombosis of an AAA presenting as Cauda equina syndrome in addition to limb ischemia has been published [10]. Furthermore, a case report of a patient with abdominal pain, AAA and a rash covering the lower part of the body in association with an ischemic Cauda equina syndrome following spinal and cutaneous embolization has also been published [11]. In our patient, the clinical presentation was different. He had a sudden, painless loss of neurological function in the lower limbs without signs of vascular compromise. The aneurysm was not clinically palpable. This kind of clinical manifestation might mislead to consider a neurologic etiology of the symptoms. Due to the patency of infrarenal aorta and iliac arteries, limb perfusion maintained adequate despite of a thick intramural thrombus within the aneurysm. There were no other embolizations except to the spinal cord that confirm the hypothesis of an embolic mechanism.

The indications for emergent treatment of the AAA were obvious. The decision to perform EVAR was based on several factors. First, the aneurysm was not totally thrombosed and it was anatomically suitable to be treated by endovascular means. Second, the operative risks would probably have been higher with an open technique compared with EVAR following longer duration of the total operation and the extended aortic occlusion time in a patient with a severe neurologic deficit. Third, the risk for further embolizations was assessed higher with invasive surgery during aortic and iliac dissections. Lastly, open surgery with revascularization of the lumbar arteries was not retained because of the risk of increasing the neurologic deficit by occlusion of the remaining lumbar 
arteries.

In the case of a sudden paralysis and paraesthesia in the lower limbs, spinal cord ischemia followed by embolization of an underlying AAA is rare, but possible causal factor even in the absence of lower limb ischemia. Modern imaging methods facilitate diagnosis in the absence of clinically detectable vascular findings. Prompt treatment is of utmost importance in such patients in order to prevent further embolizations and to regain as much neurologic function as possible.

\section{Conflict of Interest/Funding}

None

\section{References}

[1] Brunot, S., Berthier, G.V., Osseby, F., et al. (2011) Abdominal Aortic Pathology Revealed by Cauda equina Syndrome. European Neurology, 65, 198-200.

[2] Fraser, S., Roberts, L. and Murphy, E. (2009) Cauda equina Syndrome: A Literature Review of Its Definition and Clinical Presentation. Archives of Physical Medicine and Rehabilitation, 90, 1964-1968. http://dx.doi.org/10.1016/j.apmr.2009.03.021

[3] Clavert, P., Chakfé, N., Edah-Tally, S., et al. (1999) Paraplegia Secondary to Surgery of the Abdominal Aorta. Journal des Maladies Vasculaires, 24, 229-232.

[4] Eagleton, M.J. and Greenberg, R.K. (2010) Spinal and Visceral Ischemia after Endovascular Aortic Repair. The Journal of Cardiovascular Surgery (Torino), 51, 71-83.

[5] Peppelenbosch, A.G., Vermeulen Windsant, I.C., Jacobs, M.J., et al. (2010) Open Repair for Ruptured Abdominal Aortic Aneurysm and the Risk of Spinal Cord Ischemia: Review of the Literature and Risk-Factor Analysis. European Journal of Vascular and Endovascular Surgery, 40, 589-595. http://dx.doi.org/10.1016/j.ejvs.2010.07.024

[6] Dormal, P.A., Delberghe, X. and Roeland, A. (1995) Infra-Renal Aortic Aneurysm and Spinal Cord Ischaemia. Acta Chirurgica Belgica, 95, 136-138.

[7] Mallick, I.H., Kumar, S. and Samy, A. (2003) Paraplegia after Elective Repair of an Infrarenal Aortic Aneurysm. Journal of the Royal Society of Medicine, 96, 501-503. http://dx.doi.org/10.1258/jrsm.96.10.501

[8] Joo, J.B. and Cummings, A.J. (2000) Acute Thoracoabdominal Aortic Dissection Presenting as Painless, Transient Paralysis of the Lower Extremities: A Case Report. The Journal of Emergency Medicine, 19, 333-337. http://dx.doi.org/10.1016/S0736-4679(00)00264-X

[9] Latif, S., Wasti, A., Grundy, D.J., Isdale, A. and Iveson, J.M. (1995) Direct Erosion of Lumbar Spine by an Abdominal Aneurysm Resulting in Lumbar Paralysis. Paraplegia, 33, 480-481. http://dx.doi.org/10.1038/sc.1995.105

[10] Wong, S.S., Roche-Nagle, G., Oreopoulos, G., et al. (2013) Acute Thrombosis of an Abdominal Aortic Aneurysm Presenting as Cauda equina Syndrome. Journal of Vascular Surgery, 57, 218-220. http://dx.doi.org/10.1016/j.jvs.2012.06.092

[11] Fairhead, J.F., Phillips, D. and Handa, A. (2005) Embolic Spinal Cord Infarction as a Presentation of Abdominal Aortic Aneurysm. Journal of the Royal Society of Medicine, 98, 59-60. http://dx.doi.org/10.1258/jrsm.98.2.59 